\title{
EDITORIAL
}

\section{Cerebral tissue saturation, the next step in cardiopulmonary resuscitation management?}

\author{
Cornelia Genbrugge ${ }^{1,2^{*}}$, Willem Boer ${ }^{2}$, Ingrid Meex ${ }^{1,2}$, Frank Jans ${ }^{1,2}$, Jo Dens ${ }^{1,3}$ and Cathy De Deyne $e^{1,2}$
}

The goal of cardiopulmonary resuscitation (CPR) is to preserve the pre-arrest neurological state by maintaining sufficient cerebral blood flow and oxygenation, but the predictors thereof remain largely unknown. Despite recent attempts to improve the quality of basic and advanced life support, no monitored link to the neurological and physiological response of these CPR efforts has been established. The difficult decision to end pre-hospital resuscitation efforts is currently based on the circumstances of cardiac arrest, length of resuscitation efforts (if available), knowledge of pre-morbid physiological reserves, and (if present) end-tidal carbon dioxide $\left(\mathrm{ETCO}_{2}\right)$ measurement. $\mathrm{ETCO}_{2}$ is currently the only parameter proven to correlate with the likelihood of return of spontaneous circulation (ROSC), although the prediction of long-term outcome based on $\mathrm{ETCO}_{2}$ values has not been established $[1,2]$. To measure $\mathrm{ETCO}_{2}$ adequately, invasive airway management is necessary and measured values are influenced by different lung pathologies.

By using two sensors on the forehead, near infrared spectroscopy (NIRS) measures the regional difference between oxygenated and deoxygenated hemoglobin, which expresses the difference in oxygen supply and demand. It determines cerebral tissue saturation non-invasively and independent of pulsatile flow. Müllner and colleagues [3] were the first to examine the use of cerebral oxygenation in (post-)cardiac arrest circumstances. They recorded cerebral saturation in six patients with ongoing CPR in the emergency department. Patients who achieved ROSC had higher cerebral saturation values compared with the single patient without ROSC. Cerebral NIRS was also studied during coronary artery bypass surgery. A correlation between desaturation and cognitive dysfunction [4], prolonged length of hospital stay [5], and perioperative

\footnotetext{
* Correspondence: cornelia.genbrugge@uhasselt.be

${ }^{1}$ Faculty of Medicine and Life Sciences, Hasselt University, Martelarenlaan 42 Hasselt 3500, Belgium

${ }^{2}$ Department of Anesthesiology, Intensive Care, Emergency Medicine and Pain Therapy, Ziekenhuis Oost-Limburg Genk, Schiepse Bos 6, Genk 3600, Belgium

Full list of author information is available at the end of the article
}

cerebrovascular accident [6] was observed, and accordingly two landmark studies $[7,8]$ showed that a goal-directed protocol preventing cerebral desaturation resulted in a decrease in length of intensive care unit and hospital stay, lower incidence of major organ morbidity and mortality, and decreased risk of cognitive decline [4-8].

Almost 20 years after the first published study on cerebral saturation monitoring during CPR, a revival of cerebral saturation measurement during CPR is taking place. Recent published research measures cerebral saturation in patients with ongoing CPR at arrival to the emergency department, but different cerebral saturation devices and different methods for analysis of NIRS data are used. The latest research on NIRS in the CPR setting focuses on two main questions.

Firstly, can cerebral saturation values predict ROSC or neurological outcome? Ito and colleagues [9] observed higher initial cerebral saturation values for patients with a good outcome at hospital discharge and 90 days after cardiac arrest. Likewise, Parnia and colleagues [10] observed a higher mean cerebral saturation in patients achieving ROSC compared with patients without ROSC. However, this study incorporated cerebral saturation values measured at the moment of ROSC into their mean values. This could have led to an overestimation of the mean cerebral saturation values in patients achieving ROSC. In a small study, patients with ROSC had a rise in cerebral saturation during CPR in contrast to patients without ROSC [11]. No patients without an increase in cerebral saturation achieved ROSC.

The second question recently addressed is whether cerebral saturation values can guide CPR efforts. Cerebral saturation measurements are probably more useful as a dynamic measurement instead of a static, single value. Hence, low cerebral saturation values could be interpreted as a need for interventions aimed at improving cerebral oxygenation during CPR. Sustained low cerebral saturation values, despite these interventions, could indicate futile CPR efforts. It has been noted that no patients with a mean cerebral saturation of less than $30 \%$ achieved ROSC 
[12]. In contrast, patients achieving ROSC spent the majority of time during CPR with saturation values of above $30 \%$ [10]. Furthermore, patients with ROSC showed a marked increase in cerebral oxygenation throughout advanced life support compared with non-survivors. As post-cardiac arrest patients are often hemodynamically unstable the first minutes after arrest and monitoring parameters are often unreliable during transport, NIRS could fulfill an important role in this setting. Meex and colleagues [13] noticed a speedy decline in cerebral saturation when re-arrest occurred during transport. These findings are similar to those described by Frisch and colleagues [14] (tissue saturation) and in case reports analyzing cerebral saturation during transcatheter aortic valve implantation $[15,16]$.

In conclusion, preliminary data suggest that monitoring of cerebral saturation during CPR seems a likely predictor of both ROSC and neurological outcome and that it might have a role guiding CPR interventions. Although the current knowledge, obtained from small observational studies, is limited, both the further development of NIRS devices and the likely execution of well-designed large blinded observational trials, particularly in the difficult environment of out-of-hospital CPR, bode well for the future. A real-time monitoring tool providing vital information on the neurological and physiological response to CPR efforts and with predictive value for neurological outcome seems close at hand.

\section{Abbreviations \\ CPR: Cardiopulmonary resuscitation; $\mathrm{ETCO}_{2}$ : End-tidal carbon dioxide; NIRS: Near infrared spectroscopy; ROSC: Return of spontaneous circulation.}

\section{Competing interests}

The authors declare that they have no competing interests.

\section{Author details}

${ }^{1}$ Faculty of Medicine and Life Sciences, Hasselt University, Martelarenlaan 42, Hasselt 3500, Belgium. ${ }^{2}$ Department of Anesthesiology, Intensive Care, Emergency Medicine and Pain Therapy, Ziekenhuis Oost-Limburg Genk, Schiepse Bos 6, Genk 3600, Belgium. ${ }^{3}$ Department of Cardiology, Ziekenhuis Oost-Limburg Genk, Schiepse Bos 6, Genk 3600, Belgium.

Published: 1 November 2014

\section{References}

1. Touma O, Davies M: The prognostic value of end tidal carbon dioxide during cardiac arrest: a systematic review. Resuscitation 2013, 84:1470-1479.

2. Neumar RW, Otto CW, Link MS, Kronick SL, Shuster M, Callaway CW, Kudenchuk PJ, Ornato JP, McNally B, Silvers SM, Passman RS, White RD, Hess EP, Tang W, Davis D, Sinz E, Morrison L: Part 8: Adult Advanced Cardiovascular Life Support 2010 American Heart Association Guidelines for Cardiopulmonary Resuscitation and Emergency Cardiovascular Care. Circulation 2010, 122:S729-S767.

3. Müllner M, Sterz F, Binder M, Hirschl MM, Janata K, Laggner AN: Near infrared spectroscopy during and after cardiac arrest - preliminary results. Clin Intensive Care 1995, 6:107-111.

4. Yao FS, Tseng CC, Ho CY, Levin SK, Illner P: Cerebral oxygen desaturation is associated with early postoperative neuropsychological dysfunction in patients undergoing cardiac surgery. I Cardiothorac Vasc Anesth 2004, 18:552-558.
5. Edmonds $\mathrm{HL}$ : Multi-modality neurophysiologic monitoring for cardiac surgery. Heart Surg Forum 2002, 5:225-228.

6. Goldman S, Sutter F, Ferdinand F, Trace C: Optimizing intraoperative cerebral oxygen delivery using noninvasive cerebral oximetry decreases the incidence of stroke for cardiac surgical patients. Heart Surg Forum 2004, 7:E376-E381.

7. Murkin JM, Adams SJ, Novick RJ, Quantz M, Bainbridge D, Iglesias I, Cleland A, Schaefer B, Irwin B, Fox S: Monitoring brain oxygen saturation during coronary bypass surgery: a randomized, prospective study. Anesth Analg 2007, 104:51-58.

8. Slater JP, Guarino T, Stack J, Vinod K, Bustami RT, Brown JM 3rd, Rodriguez AL, Magovern CJ, Zaubler T, Freundlich K, Parr GV: Cerebral oxygen desaturation predicts cognitive decline and longer hospital stay after cardiac surgery. Ann Thorac Surg 2009, 87:36-44. discussion 44-45.

9. Ito N, Nishiyama K, Callaway CW, Orita T, Hayashida K, Arimoto H, Abe M, Endo T, Murai A, Ishikura K, Yamada N, Mizobuchi M, Anan H, Okuchi K, Yasuda H, Mochizuki T, Tsujimura Y, Nakayama T, Hatanaka T, Nagao K, for the J-POP Registry Investigators: Noninvasive regional cerebral oxygen saturation for neurological prognostication of patients with out-ofhospital cardiac arrest: a prospective multicenter observational study. Resuscitation 2014, 85:778-784.

10. Parnia S, Nasir A, Shah C, Patel R, Mani A, Richman P: A feasibility study evaluating the role of cerebral oximetry in predicting return of spontaneous circulation in cardiac arrest. Resuscitation 2012, 83:982-985.

11. Asim K, Gokhan E, Ozlem B, Ozcan Y, Deniz O, Kamil K, Murat Z, Aydın C, Selman $Y$ : An assessment of the use of near infrared spectrophotometry (cerebral oximetry) in predicting the return of spontaneous circulation in out-of-hospital cardiac arrest. Am J Emerg Med 2014, 32:14-17.

12. Ahn A, Nasir A, Malik H, D'Orazi F, Parnia S: A pilot study examining the role of regional cerebral oxygen saturation monitoring as a marker of return of spontaneous circulation in shockable (VF/VT) and non-shockable (PEA/Asystole) causes of cardiac arrest. Resuscitation 2013, 84:1713-1716.

13. Meex I, De Deyne C, Dens J, Scheyltjens S, Lathouwers K, Boer W, Vundelinckx G, Heylen R, Jans F: Feasibility of absolute cerebral tissue oxygen saturation during cardiopulmonary resuscitation. Crit Care 2013, 17:R36.

14. Frisch A, Suffoletto BP, Frank R, Martin-Gill C, Menegazzi JJ: Potential utility of near-infrared spectroscopy in out-of-hospital cardiac arrest: an illustrative case series. Prehospital Emerg Care 2012, 16:564-570.

15. Pilkington SN, Hett DA, Pierce JM, Smith DC: Auditory evoked responses and near infrared spectroscopy during cardiac arrest. Br J Anaesth 1995, 74:717-719.

16. Martens PR, Dhaese HL, Van den Brande FG, Van Laecke SM: External cardiac massage improved cerebral tissue oxygenation shown by near-infrared spectroscopy during transcatheter aortic valve implantation. Resuscitation 2010, 81:1590-1591.

doi:10.1186/s13054-014-0583-0

Cite this article as: Genbrugge et al:: Cerebral tissue saturation, the next step in cardiopulmonary resuscitation management? Critical Care 2014 18:583. 